Physics

Physics Research Publications

\title{
Brane dynamics from nonlinear realizations
}

T. E. Clark M. Nitta

T. ter Veldhuis 


\title{
Brane dynamics from nonlinear realizations
}

\author{
T. E. Clark* and Muneto Nitta ${ }^{\dagger}$ \\ Department of Physics, Purdue University, West Lafayette, Indiana 47907-1396 \\ T. ter Veldhuis \\ Department of Physics, University of Minnesota, Minneapolis, Minnesota 55455
}

(Received 26 August 2002; published 29 April 2003)

\begin{abstract}
The action for a non-BPS $p=2$ brane embedded in a flat $N=1, D=4$ target superspace is obtained through the method of nonlinear realizations of the associated super-Poincare symmetries. The brane excitation modes correspond to the Nambu-Goldstone degrees of freedom resulting from the broken space translational symmetry and the target space supersymmetries. The action for this $p=2$ brane is found to be an invariant synthesis of the Akulov-Volkov and Nambu-Goto actions. The dual D2-brane Born-Infeld action is derived. The invariant coupling of matter fields localized on the brane to the Nambu-Goldstone modes is also obtained.
\end{abstract}

DOI: 10.1103/PhysRevD.67.085026

PACS number(s): 11.27.+d, 11.30.Qc, 12.60.Jv, 14.80.Mz

\section{INTRODUCTION}

A domain wall spontaneously breaks the Poincare invariance of the target space down to the symmetry group of the world volume subspace of the wall, which includes a lower dimensional Poincaré symmetry. The long wavelength oscillation modes of the domain wall are described by the Nambu-Goldstone bosons associated with the collective coordinate translations transverse to the wall. Indeed, the Nambu-Goto action governing the zero mode fields' dynamics is easily obtained in a model independent way by nonlinearly realizing the broken symmetries on the NambuGoldstone fields $[1,2]$. In the case of a two dimensional domain wall (or $p=2$-brane) embedded in three dimensional space, the $D=3$ Poincare generators, $p^{m}$ for space-time translations and $M^{m}=\frac{1}{2} \epsilon^{m n r} M_{n r}$ for Lorentz rotations, form an unbroken subgroup $H=I S O(1,2)$ of the $D=4$ Poincare group $G=I S O(1,3)$. The broken generators are the $D=4$ translation generator transverse to the wall which is a $D$ $=3$ Lorentz scalar, denoted $Z$, and the three broken $D=4$ Lorentz rotations which form a $D=3$ Lorentz vector, denoted $K^{m}$. The $D=4$ Poincare group can be realized by group elements acting on the coset $I S O(1,3) / S O(1,2)$ element $\Omega$ formed from the $p^{m}, Z, K^{m}$ charges

$$
\Omega \equiv e^{i x^{m} p_{m}} e^{i \phi(x) Z} e^{i v^{m}(x) K_{m}}
$$

where the world volume $D=3$ space-time coordinates of the 2-brane wall in the static gauge are $x^{m}$, while $\phi(x)$ and $v^{m}(x)$ are the collective coordinate Nambu-Goldstone bosons associated with the broken $D=4$ Poincare symmetries corresponding to the excitation modes of the 2-brane. The $D=4$ Poincare group transformations are realized by left multiplication by group elements $g$,

$$
g \Omega=\Omega^{\prime} h,
$$

\footnotetext{
*Email address: clark@physics.purdue.edu

†Email address: nitta@physics.purdue.edu

${ }^{\ddagger}$ Email address: veldhuis@physics.umn.edu
}

where the new coset element $\Omega^{\prime}$ has the form

$$
\Omega^{\prime}=e^{i x^{\prime m} p_{m}} e^{i \phi{ }^{\prime}\left(x^{\prime}\right) Z} e^{i v^{\prime m}\left(x^{\prime}\right) K_{m}}
$$

and yields the transformation law for the coordinates and fields and

$$
h=e^{i \beta^{m}(g, v) M_{m}}
$$

allows $\Omega^{\prime}$ to be written as a coset element. The set of charges $\left\{p^{m}, M^{m}\right\}$ generate the vacuum stability group $H$ of the system and are linearly represented. For the general set of infinitesimal transformations $g \in G$,

$$
g=e^{i\left[a^{m} p_{m}+z Z+b^{m} K_{m}+\alpha^{m} M_{m}\right]},
$$

the $D=4$ Poincaré algebra, written in $D=3$ Lorentz group form

$$
\begin{array}{rlrl}
{\left[p^{m}, p^{n}\right]=0} & {\left[M^{m}, M^{n}\right]} & =-i \epsilon^{m n r} M_{r} \\
{\left[p^{m}, Z\right]=0} & {\left[M^{m}, K^{n}\right]} & =-i \epsilon^{m n r} K_{r} \\
{\left[K^{m}, K^{n}\right]} & =+i \epsilon^{m n r} M_{r} \\
{\left[M^{m}, p^{n}\right]} & =-i \epsilon^{m n r} p_{r} & {\left[K^{m}, p^{n}\right]} & =+i \eta^{m n} Z \\
{\left[M^{m}, Z\right]} & =0 & {\left[K^{m}, Z\right]} & =+i p^{m}
\end{array}
$$

can be exploited to find the space-time coordinate variations and field transformations

$$
\begin{aligned}
x^{\prime m}= & x^{m}+a^{m}-\phi b^{m}+\epsilon^{m n r} \alpha_{n} x_{r} \\
\Delta \phi= & z-b_{m} x^{m} \\
\Delta v^{m}= & \frac{\sqrt{v^{2}}}{\tanh \sqrt{v^{2}}} b^{m}+\left(1-\frac{\sqrt{v^{2}}}{\tanh \sqrt{v^{2}}}\right) \frac{b_{n} v^{n} v^{m}}{v^{2}} \\
& +\epsilon^{m n r} \alpha_{n} v_{r} .
\end{aligned}
$$

Here the field transformations are total variations so that $\Delta \phi(x)=\phi^{\prime}\left(x^{\prime}\right)-\phi(x)$, and likewise for $v^{m}$.

Constructing the Maurer-Cartan world volume one-forms, 


$$
\Omega^{-1} d \Omega \equiv i\left[\omega^{a} p_{a}+\omega_{Z} Z+\omega_{K}^{a} K_{a}+\omega_{M}^{a} M_{a}\right],
$$

defines the dreibein, $e_{m}{ }^{a}$, which relates the covariant world volume coordinate differentials $\omega^{a}$ to the world volume coordinate differentials $d x^{m}$, so that $\omega^{a}=d x^{m} e_{m}{ }^{a}$, the covariant derivatives of the fields, $\omega_{Z} \equiv \omega^{a} \nabla_{a} \phi$ and $\omega_{K}^{b} \equiv \omega^{a} \nabla_{a} v^{b}$, and the spin connection $\omega_{M}^{b} \equiv \omega^{a} \Gamma_{a}{ }^{b}$. Once again utilizing the $D=4$ Poincare algebra, the dreibein is found to be

$$
e_{m}{ }^{a}=\delta_{m}{ }^{a}+\left[\cosh \sqrt{v^{2}}-1\right] \frac{v_{m} v^{a}}{v^{2}}+\partial_{m} \phi v^{a} \frac{\sinh \sqrt{v^{2}}}{\sqrt{v^{2}}}
$$

while the $\phi$-field covariant derivative is

$$
\begin{aligned}
\omega_{Z}= & \omega^{a} \nabla_{a} \phi=d x^{m} e_{m}{ }^{a} \nabla_{a} \phi \\
= & d x^{m} \cosh \sqrt{v^{2}} \\
& \times\left[\partial_{m} \phi+v_{m} \frac{\tanh \sqrt{v^{2}}}{\sqrt{v^{2}}}\right] .
\end{aligned}
$$

The one-form transformation laws follow from Eq. (1.2),

$$
\left(\Omega^{-1} d \Omega\right)^{\prime}=h\left(\Omega^{-1} d \Omega\right) h^{-1}+h d h^{-1},
$$

and are homogeneous except for the case of the broken $D$ $=4$ Lorentz rotations generated by $K^{n}$, in which case

$$
h=e^{-(i / 2)\left[\tanh (1 / 2) \sqrt{v^{2}} /(1 / 2) \sqrt{v^{2}}\right] b_{m} v_{r} \epsilon^{m r n} M_{n},}
$$

implying that $\omega_{M}^{m}$ transforms with an additional inhomogeneous term as required of a connection one-form.

Given these building blocks and their transformation laws, the low energy $G$-invariant action, $\Gamma$, is obtained in leading order in the domain wall (brane)tension $\sigma$,

$$
\Gamma=-\sigma \int d^{3} x \operatorname{det} e,
$$

with the determinant of $e$ determined to be

$$
\operatorname{det} e=\cosh \sqrt{v^{2}}\left[1+\partial_{n} \phi v^{n} \frac{\tanh \sqrt{v^{2}}}{\sqrt{v^{2}}}\right] .
$$

Since the dreibein depends only on $v^{m}$ and not its derivatives, its Euler-Lagrange equation of motion can be used to eliminate $v^{m}$ in terms of $\phi$. This is just the "inverse Higgs mechanism" [3], equivalently obtained by setting the $\phi$ covariant derivative, Eq. (1.10), to zero: $\nabla_{a} \phi=0$ and hence

$$
v^{m} \frac{\tanh \sqrt{v^{2}}}{\sqrt{v^{2}}}=-\partial^{m} \phi .
$$

Substituting this into the dreibein, it has the form

$$
\begin{aligned}
e_{m}{ }^{a} & =\delta_{m}{ }^{a}+\left(\frac{1-\cosh \sqrt{v^{2}}}{\cosh \sqrt{v^{2}}}\right) \frac{v_{m} v^{a}}{v^{2}} \\
& =\delta_{m}{ }^{a}-\left(1-\sqrt{1-(\partial \phi)^{2}}\right) \frac{\partial_{m} \phi \partial^{a} \phi}{(\partial \phi)^{2}} .
\end{aligned}
$$

The determinant of $e$ simplifies to become

$$
\operatorname{det} e=\frac{1}{\cosh \sqrt{v^{2}}}=\sqrt{1-\partial_{m} \phi \partial^{m} \phi},
$$

and the Nambu-Goto action [4-6] for a $p=2$ brane embedded in $D=4$ space-time (in the static gauge)is obtained

$$
\Gamma=-\sigma \int d^{3} x \sqrt{1-\partial_{m} \phi \partial^{m} \phi}
$$

Alternatively, the $\phi$ and $v^{m}$ fields can be kept as independent degrees of freedom. The action is given in terms of Eq. (1.14). The $\phi$ equation of motion, $\delta \Gamma / \delta \phi=0$, can be expressed as the $D=3$ Bianchi identity, $\partial_{m} F^{m}=0$, for the field strength vector

$$
F^{m} \equiv v^{m} \frac{\sinh \sqrt{v^{2}}}{\sqrt{v^{2}}} .
$$

Substituting this into Eq. (1.14) yields $\operatorname{det} e=\sqrt{1+F^{2}}$ $+\partial_{m} \phi F^{m}$. Integrating the second term by parts and using $\partial_{m} F^{m}=0$ implies duality of the Nambu-Goto $p=2$ brane action to the Born-Infeld action [7] for a D2-brane

$$
\Gamma=-\sigma \int d^{3} x \operatorname{det} e=-\sigma \int d^{3} x \sqrt{1+F^{2}} .
$$

A slightly generalized approach can be applied to the above coset method as described in [8]. The brane world volume is parametrized by the $D=3$ vector $\xi^{m}$ and the brane's generalized coordinates are maps of this $D=3$ parameter space into the $D=4$ target manifold: $x^{\mu}(\xi)=\left(x^{m}(\xi), \phi(\xi)\right)$. The exterior derivative is given by $d=d \xi^{m} \partial / \partial \xi^{m}$ and it is world volume reparametrization invariant. The Maurer-Cartan oneforms, Eq.( 1.8), are also reparametrization invariant since they depend on the exterior derivative. From this point of view the covariant differential one-forms, $\omega^{a}$, define the dreibein as $\omega^{a} \equiv d \xi^{m} e_{m}{ }^{a}$ where now

$$
e_{m}{ }^{a}=\frac{\partial x^{a}}{\partial \xi^{m}}+\left[\cosh \sqrt{v^{2}}-1\right] \frac{v_{b} \frac{\partial x^{b}}{\partial \xi^{m}} v^{a}}{v^{2}}+\frac{\partial \phi}{\partial \xi^{m}} v^{a} \frac{\sinh \sqrt{v^{2}}}{\sqrt{v^{2}}} .
$$

Similarly the covariant differential one-form $\omega_{Z}$ of the $\phi(\xi)$ coordinate is given by its covariant derivative 


$$
\omega_{Z}=\omega^{a} \nabla_{a} \phi=d \xi^{m} \cosh \sqrt{v^{2}}\left[\frac{\partial \phi}{\partial \xi^{m}}+\frac{\partial x^{a}}{\partial \xi^{m}} v_{a} \frac{\tanh \sqrt{v^{2}}}{\sqrt{v^{2}}}\right] .
$$

The Maurer-Cartan one-form can be calculated sequentially as

$$
\begin{aligned}
\Omega^{-1} d \Omega & =e^{-i v^{n} K_{n}}\left(d+i d x^{\mu} P_{\mu}\right) e^{+i v^{n} K_{n}} \\
& =i d x^{\mu} \Lambda_{\mu}{ }^{\nu}(v) P_{\nu}+e^{-i v^{n} K_{n}} d e^{+i v^{n} K_{n}},
\end{aligned}
$$

where $\Lambda_{\mu}{ }^{\nu}(v)$ is a broken $D=4$ Lorentz transformation determined by $v^{n}$, and $P_{\mu}=\left(p_{m}, Z\right)$. Thus it is seen that the one-forms $\omega^{\mu} \equiv\left(\omega^{a}, \omega_{Z}\right)$ are related to $d x^{\mu}$ by a Lorentz transformation

$$
\omega^{\mu}=d x^{\nu} \Lambda_{\nu}{ }^{\mu}(v) .
$$

From the invariance of the $D=4$ Minkowski metric $\eta_{\mu \nu}$ under $D=4$ Lorentz transformations, it follows that

$$
\begin{aligned}
\omega^{\mu} \eta_{\mu \nu} \omega^{\nu} & =d x^{\mu} \Lambda_{\mu}{ }^{\rho} \eta_{\rho \sigma} \Lambda_{\nu}{ }^{\sigma} d x^{\nu} \\
& =d x^{\mu} \eta_{\mu \nu} d x^{\nu} .
\end{aligned}
$$

As before, the inverse Higgs mechanism may be applied, $\omega_{Z}=0$, yielding

$$
\begin{aligned}
\omega^{\mu} \eta_{\mu \nu} \omega^{\nu} & =d \xi^{m} e_{m}{ }^{a} \eta_{a b} e_{n}{ }^{b} d \xi^{n} \\
& =d \xi^{m} \frac{\partial x^{\mu}}{\partial \xi^{m}} \eta_{\mu \nu} \frac{\partial x^{\nu}}{\partial \xi^{n}} d \xi^{n} .
\end{aligned}
$$

This is just the $G$-invariant interval, hence the world volume reparametrization invariant and $G$-invariant action is

$$
\Gamma=-\sigma \int d^{3} \xi \operatorname{det} e=-\sigma \int d^{3} \xi \sqrt{\operatorname{det}\left(\frac{\partial x^{\mu}}{\partial \xi^{m}} \eta_{\mu \nu} \frac{\partial x^{\nu}}{\partial \xi^{n}}\right)} .
$$

Thus, the general form of the Nambu-Goto action for a $p$ $=2$ brane is secured. The reparametrization invariance may be used to fix the static gauge: $x^{m}=\xi^{m}$ and $\phi=\phi(x)$, in which case the action reduces to that of Eq. (1.18). The remainder of the paper is in the static gauge.

The above considerations can be generalized to apply in a supersymmetric context by embedding a topological defect in superspace. Apart from Goldstone bosons associated with spontaneously broken translational invariances, there are in this case additional fermionic long wavelength oscillations. These Goldstinos reflect collective Grassmann coordinates which are associated with spontaneously broken supersymmetries. Additional massless world volume degrees of freedom may be required to complete multiplets of the unbroken supersymmetries. Topological defects which spontaneously break down target space super-Poincaré invariance to a lower dimensional super-Poincaré symmetry were considered by $[9,10]$. If the spatial extension of a defect in directions of broken translational invariance is small compared to the wavelength of its fluctuations, and if in addition some supersymmetry remains unbroken, then such a defect is a super $p$-brane $[11,12]$. The world volume theory on the defect inherits extended supersymmetry. Part of this supersymmetry as well as central charges corresponding to spontaneously broken translation generators of the target space are nonlinearly realized [13].

The previous illustrative example dealt with a $D=3$ space-time world volume of the $p=2$ brane being embedded in a target $D=4$ space-time. Alternatively, the $D=3$ spacetime world volume can be embedded in a target $N=1, D$ $=4$ superspace; this is the case of a non-Bogemol'nyiPrasad-Sommerfield saturated (non-BPS) brane embedded into $N=1, D=4$ superspace, the main topic of this paper. When such a 2-brane domain wall is embedded into superspace, all supersymmetry is spontaneously broken as well as the spatial translation symmetry.

If in contrast the defect is a BPS domain wall, then the supersymmetry is only partially broken [14]. The tension saturates its lower bound, which is equal to the absolute value of the central charge, and the domain wall is therefore stable. The world volume of the corresponding super 2-brane is $N=1, D=3$ superspace. In the thin wall limit its dynamics were studied using the method of nonlinear realizations $[15,16]$ as well as equivalently using the superembedding technique [17]. The world volume theory of BPS domain walls with finite width was also studied $[18,19]$ through an expansion in modes about classical domain wall solutions.

BPS saturated domain walls provide an effective mechanism for the partial breaking of supersymmetry and may even be a necessary ingredient in a more fundamental brane world and M-theory description of nature. On the other hand, a non-BPS domain wall can be stable and as such can provide a means to completely break the supersymmetry. The lower dimensional manifold of the domain wall will then also include the corresponding Goldstino modes besides the broken translational symmetry Nambu-Goldstone boson mode. These fields correspond to the excitations of the brane in all possible target space directions, the space direction orthogonal to the brane and in this case the Grassmann coordinate directions $\theta_{\alpha}$ and $\bar{\theta}_{\dot{\alpha}}$ of $N=1, D=4$ superspace when in the static gauge. It is the purpose of this paper to construct the effective action via the method of nonlinear realizations for these low energy degrees of freedom. In addition to the massless Nambu-Goldstone fields of the 2-brane motion, there also can be light matter field degrees of freedom localized on the domain wall brane. Their invariant interaction with the Nambu-Goldstone fields is determined as well.

Section II analyzes the method of the nonlinear realization of $N=1, D=4$ super-Poincare symmetries on the NambuGoldstone fields as coset manifold coordinates. The associated Maurer-Cartan one-forms are constructed in Sec. III. Included in these is the $D=3$ world volume dreibein which is used to construct the $N=1, D=4$ super-Poincare invariant action. In Sec. IV, the covariant derivatives of the NambuGoldstone fields, obtained from the Maurer-Cartan oneforms, are shown to provide a means to covariantly reduce 
the number of fields, through the inverse Higgs mechanism [3], to the minimal set of independent degrees of freedom needed to describe the fluctuations of the 2-brane in $N$ $=1, D=4$ superspace. This description of the brane dynamics is performed in the partially covariant one-form basis which reveals the product nature of the world volume dreibein and leads directly to the invariant synthesis of the Nambu-Goto and Akulov-Volkov actions. Alternatively, exploiting the general form of the brane action in terms of all fields, the duality between the Nambu-Goto-Akulov-Volkov action and the $D 2$-brane (nonlinearly realized) supersymmetric Born-Infeld action is derived. Finally, in Sec. V, the invariant action describing scalar and fermion matter fields localized on the brane is constructed.

The brane and matter field actions are the lowest order terms in an expansion in powers of the domain wall thickness. In this approximation the 2-brane is thin relative to its fluctuation wavelength, but the amplitude of the fluctuations may be large. The covariant derivatives for Goldstone and matter fields determined in Secs. IV and V form the building blocks from which higher order terms in the expansion can be constructed in order to obtain an action that describes large amplitude, shorter wavelength (but still larger than the domain wall thickness) fluctuations as well. Additional higher order terms have coefficients that parametrize in the world volume theory indirect effects of massive modes which exist in the underlying fundamental theory. Such massive modes generically have masses proportional to the inverse of the domain wall width. The remainder of the Introduction outlines the results derived in Secs. II-V of the body of the paper.

The Nambu-Goldstone modes' action is an invariant synthesis of the Akulov-Volkov action [20] and the Nambu-Goto action. This action consists of a product of the AkulovVolkov lagrangian and a modified Nambu-Goto Lagrangian allowing for excitations of the non-BPS brane in the Grassmann coordinate directions of the target superspace

$$
\Gamma=-\sigma \int d^{3} x \operatorname{det} e=-\sigma \int d^{3} x \operatorname{det} \hat{e} \operatorname{det} N
$$

where the 2-brane dreibein is given by a product of dreibeine $e_{m}{ }^{a}=\hat{e}_{m}{ }^{b} N_{b}{ }^{a}$. The Akulov-Volkov dreibein $\hat{e}_{m}{ }^{a}$ is

$$
\hat{e}_{m}{ }^{a}=A_{m}{ }^{a}=\delta_{m}{ }^{a}+i \partial_{m} \theta \gamma^{0} \gamma^{a} \theta+i \partial_{m} \lambda \gamma^{0} \gamma^{a} \lambda,
$$

with the Goldstino fields given by the $D=3$ Majorana spinors $\theta_{i}(x)$ and $\lambda_{i}(x)$. The Akulov-Volkov determinant term in the action has its typical form

$$
\operatorname{det} \hat{e}=\operatorname{det}\left[\delta_{m}{ }^{a}+i \partial_{m} \theta \gamma^{0} \gamma^{a} \theta+i \partial_{m} \lambda \gamma^{0} \gamma^{a} \lambda\right]
$$

The Nambu-Goto dreibein $N_{a}{ }^{b}$ is given by a supersymmetric extension of Eq. (1.9) above

$$
\begin{aligned}
N_{a}{ }^{b}= & \delta_{a}{ }^{b}+\left[\cosh \sqrt{v^{2}}-1\right] \frac{v_{a} v^{b}}{v^{2}}+\left(\hat{\mathcal{D}}_{a} \phi+\hat{\mathcal{D}}_{a} \theta \gamma^{0} \lambda\right. \\
& \left.-\theta \gamma^{0} \hat{\mathcal{D}}_{a} \lambda\right) v \frac{\sinh \sqrt{v^{2}}}{\sqrt{v^{2}}}
\end{aligned}
$$

where $\hat{\mathcal{D}}_{a}=\hat{e}_{a}^{-1 m} \partial_{m}$ is the Akulov-Volkov partial covariant derivative. The determinant of the Nambu-Goto dreibein is found to be

$$
\begin{aligned}
\operatorname{det} N= & \cosh \sqrt{v^{2}}\left[1+\left(\hat{\mathcal{D}}_{a} \phi+\hat{\mathcal{D}}_{a} \theta \gamma^{0} \lambda\right.\right. \\
& \left.\left.-\theta \gamma^{0} \hat{\mathcal{D}}_{a} \lambda\right) v^{a} \frac{\tanh \sqrt{v^{2}}}{\sqrt{v^{2}}}\right] .
\end{aligned}
$$

As with the dreibein, the Maurer-Cartan one-form associated with the central charge $Z$ has a supersymmetric generalization to include motion in the anticommuting directions

$$
\begin{aligned}
\omega_{Z}= & \cosh \sqrt{v^{2}}\left[\left(d \phi+d \theta \gamma^{0} \lambda-\theta \gamma^{0} d \lambda\right)\right. \\
& \left.+d x^{m} \hat{e}_{m}{ }^{a} v_{a} \frac{\tanh \sqrt{v^{2}}}{\sqrt{v^{2}}}\right]
\end{aligned}
$$

Setting it to zero once again leads to the "inverse Higgs mechanism:"

$$
v_{a} \frac{\tanh \sqrt{v^{2}}}{\sqrt{v^{2}}}=-\left(\hat{\mathcal{D}}_{a} \phi+\hat{\mathcal{D}}_{a} \theta \gamma^{0} \lambda-\theta \gamma^{0} \hat{\mathcal{D}}_{a} \lambda\right)
$$

Thus the super Nambu-Goto determinant reduces to

$$
\operatorname{det} N=\frac{1}{\cosh \sqrt{v^{2}}}=\sqrt{1-\left(\hat{\mathcal{D}}_{a} \phi+\hat{\mathcal{D}}_{a} \theta \gamma^{0} \lambda-\theta \gamma^{0} \hat{\mathcal{D}}_{a} \lambda\right)^{2}} .
$$

Hence, the $D=4$ super-Poincare invariant action is obtained from the product of Eqs. (1.30) and (1.35).

The domain wall world volume embedded in superspace is dual to the $D 2$-brane embedded in superspace $[21,22]$ as is expressed by the above Nambu-Goto-Akulov-Volkov action being dual to the supersymmetric Born-Infeld action. Treating all fields as independent degrees of freedom, the $\phi$ field equation is obtained from Eqs. (1.30) and (1.32) above and takes the form of the $D=3$ Bianchi identity, $\partial_{m} F^{m}=0$, with the field strength vector now given by

$$
F^{m} \equiv \operatorname{det} \hat{e} \frac{\sinh \sqrt{v^{2}}}{\sqrt{v^{2}}} v^{a} \hat{e}_{a}^{-1 m} .
$$

Substituting this into the determinant of the dreibein yields 


$$
\operatorname{det} \hat{e} \operatorname{det} N=\operatorname{det} \hat{e} \cosh \sqrt{v^{2}}+F^{n}\left(\partial_{n} \theta \gamma^{0} \lambda-\theta \gamma^{0} \partial_{n} \lambda\right)+F^{n} \partial_{n} \phi .
$$

The last term integrates to zero to obtain that the non-BPS $p=2$ brane Nambu-Goto-Akulov-Volkov action is dual to the D2-brane supersymmetric Born-Infeld action

$$
\begin{aligned}
\Gamma & =-\sigma \int d^{3} x\left(\sqrt{\operatorname{det}\left(\hat{g}_{m n}+F_{m n}\right)}+F^{n}\left[\partial_{n} \theta \gamma^{0} \lambda-\theta \gamma^{0} \partial_{n} \lambda\right]\right) \\
& =-\sigma \int d^{3} x\left(\sqrt{\operatorname{det} \hat{g}+F^{m} \hat{g}_{m n} F^{n}}+F^{n}\left[\partial_{n} \theta \gamma^{0} \lambda-\theta \gamma^{0} \partial_{n} \lambda\right]\right),
\end{aligned}
$$

where the Akulov-Volkov metric is given by $\hat{g}_{m n}$ $=\hat{e}_{m}{ }^{a} \eta_{a b} \hat{e}_{n}{ }^{b}$ and $F_{m n}=\epsilon_{m n r} F^{r}$.

A specific example of an underlying field theory realizing a stable non-BPS domain wall can be constructed as a generalized Wess-Zumino model in $D=4$ dimensions [18]. It contains two chiral superfields $X$ and $\Phi$, with superpotential $W=X\left(\mu^{2}-\lambda \Phi^{2}\right)$ and canonical Kähler potential. The tension $\sigma=\frac{8}{3} \mu^{3} / \sqrt{\lambda}$ of the domain wall that interpolates between the two vacua $X=0, \Phi= \pm \sqrt{\mu^{2} / \lambda}$ does not saturate the BPS bound $|2 \Delta W|=0$, yet the wall is stable. The width of the wall is $1 / \sqrt{\mu^{2} \lambda}$. All of the supersymmetry and the translational symmetry in one direction are broken by the domain wall solution, however the $R$ symmetry of the model is left unbroken. The quantum fluctuations about the wall solution include the zero mode Nambu-Goldstone and Goldstino excitations. The spectrum in addition contains a number of localized massive excitations corresponding to breathing modes of the wall with masses between $\sqrt{\mu^{2} \lambda}$ and $2 \sqrt{\mu^{2} \lambda}$, and a continuum of nonlocalized modes starting at $2 \sqrt{\mu^{2} \lambda}$. The parameters of the effective domain wall world volume theory valid below the scale $\sqrt{\mu^{2} \lambda}$ of all the massive modes are in principle determined by integrating out these massive excitations. However, the form of the low energy effective action is determined solely by the group theoretical nonlinearly realized broken symmetry techniques discussed above. The thin domain wall action is given by Eqs. (1.28), (1.30) and (1.35). Non-BPS domain walls also have been considered in case one of the target space dimensions is compact [23].

The brane localized matter fields' action is constructed using the covariant derivatives of the nonlinearly realized spontaneously broken $D=4$ super-Poincaré symmetries. To this end the method to include matter fields in theories with nonlinearly realized supersymmetry [24] is extended to also include nonlinearly realized translation symmetry. The form of the leading terms in the domain wall width expansion of the effective action for scalar, $S(x)$, and fermion, $\psi_{i}(x)$, matter fields is determined to be

$$
\Gamma_{\text {matter }}=\int d^{3} x \operatorname{det} e \mathcal{L}_{\text {matter }}
$$

with the $G$-invariant matter field Lagrangian

$$
\mathcal{L}_{\text {matter }}=\nabla_{a} S \eta^{a b} \nabla_{b} S-V(S)+i \bar{\psi} \gamma^{a} \nabla_{a} \psi-m \bar{\psi} \psi+Y(S, \bar{\psi} \psi)
$$

The scalar field potential $V(S)$ is an arbitrary function of $S$ and the generalized Yukawa coupling $Y(S, \bar{\psi} \psi)$ is a function coupling the scalar fields $S$ to the scalar bilinears $\bar{\psi} \psi$. In the case of a single species of $D=3$ Majorana fermion, the Yukawa term terminates at the form $y(S) \bar{\psi} \psi$, with the arbitrary Yukawa coupling function $y(S)$. The masses and coupling constants of the matter are left as parameters of the effective theory to be specified by the matching to a specific underlying domain wall model. The $G$-covariant derivatives of the matter fields are obtained in terms of the $G$-covariant space-time derivatives $\mathcal{D}_{a}=e_{a}^{-1 m} \partial_{m}$ and the components of the spin connection $\omega_{M}^{b}=\omega^{a} \Gamma_{a}^{b}$ in the $G$-covariant basis $\omega^{a}=d x^{m} e_{m}{ }^{a}$

$$
\begin{aligned}
\nabla_{a} S & =\mathcal{D}_{a} S \\
\nabla_{a} \psi_{i} & =\mathcal{D}_{a} \psi_{i}-\frac{i}{2} \Gamma_{a}{ }^{b} \gamma_{b i j} \psi_{j} .
\end{aligned}
$$

The fully covariant derivatives $\mathcal{D}_{a}$ can be expressed in terms of the partially covariant Akulov-Volkov derivatives $\hat{\mathcal{D}}_{a}$ with the help of the Nambu-Goto dreibein

$$
\mathcal{D}_{a}=N_{a}^{-1 b} \hat{\mathcal{D}}_{b} .
$$

Likewise, the components of the spin connection can be expressed in this partially covariant basis $\Gamma_{a}{ }^{b}=N_{a}^{-1 c} \hat{\Gamma}_{c}{ }^{b}$. So doing, the fully $G$-invariant matter field Lagrangian in the partially covariant basis becomes

$$
\begin{aligned}
\mathcal{L}_{\text {matter }}= & \hat{\nabla}_{a} S n^{a b} \hat{\nabla}_{b} S-V(S)+i \bar{\psi} \gamma^{b} N_{b}^{-1 a} \hat{\nabla}_{a} \psi-m \bar{\psi} \psi \\
& +Y(S, \bar{\psi} \psi),
\end{aligned}
$$

with the Nambu-Goto metric given in terms of the NambuGoto dreibein $n^{a b}=N_{c}^{-1 a} \eta^{c d} N_{d}^{-1 b}$ while the partially covariant matter field derivatives are defined by

$$
\begin{aligned}
\hat{\nabla}_{a} S & =\hat{\mathcal{D}}_{a} S \\
\hat{\nabla}_{a} \psi_{i} & =\hat{\mathcal{D}}_{a} \psi_{i}-\frac{i}{2} \hat{\Gamma}_{a}{ }^{n} \gamma_{n i j} \psi_{j} .
\end{aligned}
$$

Finally, the Appendix is a summary of $\mathrm{D}=3$ Lorentz spinor and tensor definitions and identities along with Dirac matrix conventions.

\section{COSET CONSTRUCTION AND SUPER-POINCARÉ SYMMETRIES}

Besides the space-time translation and Lorentz rotation generators, the $N=1, D=4$ super-Poincare transformations include the Weyl spinor supersymmetry charges $Q_{\alpha}$ and $\bar{Q}_{\alpha}$ obeying the anticommutation relation 


$$
\left\{Q_{\alpha}, \bar{Q}_{\dot{\alpha}}\right\}=+2 \sigma_{\alpha \dot{\alpha}}^{\mu} P_{\mu} .
$$

This relation is invariant under the automorphism generated by the $R$ charge

$$
\begin{aligned}
& {\left[R, Q_{\alpha}\right]=+Q_{\alpha}} \\
& {\left[R, \bar{Q}_{\dot{\alpha}}\right]=-\bar{Q}_{\dot{\alpha}}} \\
& {\left[R, P^{\mu}\right]=0=\left[R, M^{\mu \nu}\right] .}
\end{aligned}
$$

A domain wall spontaneously breaks the $D=4$ symmetries to those of $D=3$. In the case of a static planar non-BPS domain wall centered on the $x-y$ plane, the above symmetries are broken to only retain those of the $D=3$ Poincare transformations of the wall. Nambu-Goldstone zero mode degrees of freedom corresponding to the broken $z$ direction translation generator and the four supersymmetry generators propagate along the wall. Geometrically this describes the embedding of a non-BPS spatial 2-brane having a $D=3$ space-time world volume into a target $N=1, D=4$ superspace. In the static gauge, the Nambu-Goldstone boson describes motion of the brane in the spatial $(z)$ direction normal to the brane while the Goldstino fields correspond to motion of the brane in the Grassmann coordinate directions of $N=1, D=4$ superspace.

Since the unbroken symmetries are those of the $D=3$ Poincare group, it is useful to express the $D=4$ charges in terms of their $D=3$ Lorentz group transformation properties. However, the SUSY is completely broken in the non-BPS case, so the fields will not belong to linear SUSY representation multiplets. Thus, the space-time translation generator $P^{\mu}$, which transforms as a vector $\left(\frac{1}{2}, \frac{1}{2}\right)$ representation of the $D=4$ Lorentz group, consists of a $D=3$ Lorentz group vector, $p^{m}=P^{m}$, with $m=0,1,2$, and a $D=3$ central charge scalar, $Z \equiv P_{3}$. Likewise, the Lorentz transformation charges $M^{\mu \nu}$ are in the $D=4(1,1)_{A}$ representation which consists of two $D=3$ vector representations: $M^{m n}=\epsilon^{m n r} M_{r}$ and $K^{m}$ $\equiv M^{m 3}$. The $R$ charge is a singlet from both points of view. Finally the $D=4 \operatorname{SUSY}\left(\frac{1}{2}, 0\right)$ spinor $Q_{\alpha}$ and the $\left(0, \frac{1}{2}\right)$ spinor $\bar{Q}_{\dot{\alpha}}$ consist of two $D=3$ two-component Majorana spinors: $q_{i}$ and $s_{i}$, with $i=1,2$, comprising the charges for (centrally extended) $N=2, D=3$ SUSY. These spinors are given as linear combinations of $Q_{\alpha}$ and $\bar{Q}_{\dot{\alpha}}$ according to

$$
\begin{aligned}
& q_{1}=\frac{1}{2}\left[a\left(Q_{1}-Q_{2}\right)+\bar{a}\left(\bar{Q}_{1}-\bar{Q}_{2}\right)\right] \\
& q_{2}=\frac{-i}{2}\left[a\left(Q_{1}+Q_{2}\right)-\bar{a}\left(\bar{Q}_{1}+\bar{Q}_{2}\right)\right] \\
& s_{1}=\frac{1}{2}\left[\bar{a}\left(Q_{1}-Q_{2}\right)+a\left(\bar{Q}_{1}-\bar{Q}_{2}\right)\right] \\
& s_{2}=\frac{-i}{2}\left[\bar{a}\left(Q_{1}+Q_{2}\right)-a\left(\bar{Q}_{1}+\bar{Q}_{2}\right)\right],
\end{aligned}
$$

where the complex number $a$ is $a \equiv e^{i \pi / 4}$.

The $N=1, D=4$ super-Poincare algebra can be written in terms of the $D=3$ Lorentz group representation charges as that in Eq. (1.6) and the commutators involving the supersymmetry charges

$$
\begin{array}{ll}
{\left[M^{m n}, q_{i}\right]=-\frac{1}{2} \gamma_{i j}^{m n} q_{j}} & {\left[K^{m}, q_{i}\right]=+\frac{1}{2} \gamma_{i j}^{m} s_{j}} \\
{\left[M^{m n}, s_{i}\right]=-\frac{1}{2} \gamma_{i j}^{m n} s_{j}} & {\left[K^{m}, s_{i}\right]=-\frac{1}{2} \gamma_{i j}^{m} q_{j}} \\
{\left[R, q_{i}\right]=+i s_{i}} & \left\{q_{i}, q_{j}\right\}=+2\left(\gamma^{m} C\right)_{i j} p_{m} \\
{\left[R, s_{i}\right]=-i q_{i}} & \left\{s_{i}, s_{j}\right\}=+2\left(\gamma^{m} C\right)_{i j} p_{m} \\
& \left\{q_{i}, s_{j}\right\}=-2 i C_{i j} Z .
\end{array}
$$

The charge conjugation matrix and the $2+1(D=3)$ dimensional gamma matrices in the appropriate representation are presented in the Appendix.

The action for the 2-brane can be found by means of the coset construction. Towards this end a coset $G / S O(1,2) \otimes R$ element $\Omega$, with $G$ the $N=1, D=4$ super-Poincaré group, is written as

$$
\Omega \equiv e^{i x^{m} p_{m}} e^{i\left[\phi Z+\bar{\theta}_{i} q_{i}+\bar{\lambda}_{i} s_{i}\right]} e^{i v^{m} K_{m}}
$$

where the $x^{m}$ denote the $D=3$ space-time coordinates parametrizing the world volume of the 2-brane in the static gauge, while the Nambu-Goldstone fields, denoted by $\phi(x), \theta_{i}(x), \lambda_{i}(x)$ and $v^{m}(x)$, describe the target space excitations of the brane. Taken together, they act as coordinates of the coset manifold. The unbroken symmetry group $H$ is generated by the set of charges $\left\{p^{m}, M^{m}, R\right\}$. Multiplication of the coset elements $\Omega$ by group elements $g \in G$ from the left results in transformations of the space-time coordinates and the Nambu-Goldstone fields according to the general structure

$$
g \Omega=\Omega^{\prime} h,
$$

where the infinitesimal transformations $g$ are parametrized as

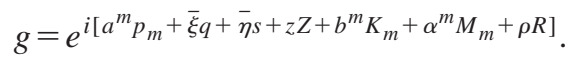

Upon application of the Baker-Campbell-Hausdorff formulas for infinitesimal $A$ and arbitrary $B$, with Lie derivative $\mathcal{L}_{A} \cdot B=[A, B]$,

$$
\begin{aligned}
e^{A} e^{B} e^{-A} & =e^{B+[A, B]} \\
e^{B} e^{A} & =e^{B+\mathcal{L}_{B / 2} \cdot\left[A+\operatorname{coth}\left(\mathcal{L}_{B / 2}\right) \cdot A\right]} \\
e^{A} e^{B} & =e^{B-\mathcal{L}_{B / 2} \cdot\left[A-\operatorname{coth}\left(\mathcal{L}_{B / 2}\right) \cdot A\right]},
\end{aligned}
$$

the transformed coset element is given by the total variation of the fields so that 


$$
\Omega^{\prime}=e^{i x^{\prime m} p_{m}} e^{i\left[\phi^{\prime}\left(x^{\prime}\right) Z+\bar{\theta}_{i}^{\prime}\left(x^{\prime}\right) q_{i}+\bar{\lambda}_{i}^{\prime}\left(x^{\prime}\right) s_{i}\right]} e^{i v^{\prime n}\left(x^{\prime}\right) K_{n},}
$$

while $h$ allows $\Omega^{\prime}$ to be written as a coset element and is given by

$$
\begin{aligned}
h & =e^{i\left(\alpha^{n} M_{m}+\rho R-(1 / 2)\left[\tanh (1 / 2) \sqrt{v^{2}} /(1 / 2) \sqrt{v^{2}}\right] b_{m} v_{r} \epsilon^{m r n} M_{n}\right)} \\
& =e^{i \rho R} e^{i \beta^{m}(g, v) M_{m}} .
\end{aligned}
$$

The infinitesimal transformations induced on the 2-brane space-time coordinates and fields are obtained as

$$
\begin{aligned}
& x^{\prime m}= x^{m}+a^{m}-i\left(\bar{\xi} \gamma^{m} \theta+\bar{\eta} \gamma^{m} \lambda\right)-\phi b^{m}+\epsilon^{m n r} \alpha_{n} x_{r} \\
& \Delta \theta_{i}= \xi_{i}+\frac{i}{2} b_{m}\left(\gamma^{m} \lambda\right)_{i}-i \rho \lambda_{i}-\frac{i}{2} \alpha_{m}\left(\gamma^{m} \theta\right)_{i} \\
& \Delta \lambda_{i}=\eta_{i}-\frac{i}{2} b_{m}\left(\gamma^{m} \theta\right)_{i}+i \rho \theta_{i}-\frac{i}{2} \alpha_{m}\left(\gamma^{m} \lambda\right)_{i} \\
& \Delta \phi=z+\left(\xi \gamma^{0} \lambda-\theta \gamma^{0} \eta\right)-b^{m} x_{m} \\
& \Delta v^{m}=+\frac{\sqrt{v^{2}}}{\tanh \sqrt{v^{2}}}\left(b^{m}-\frac{v^{r} b_{r} v^{m}}{v^{2}}\right) \\
&+\frac{v^{r} b_{r} v^{m}}{v^{2}}+\epsilon^{m n r} \alpha_{n} v_{r} .
\end{aligned}
$$

The intrinsic variation of the fields, $\delta \varphi \equiv \varphi^{\prime}(x)-\varphi(x)$, is related to the above total variation, $\Delta \varphi$, by the Taylor expansion shift in the space-time coordinates:

$$
\delta \varphi=\Delta \varphi-\delta x^{m} \partial_{m} \varphi,
$$

with $\delta x^{m}=x^{\prime m}-x^{m}$.

The nonlinearly realized $D=4$ super-Poincaré symmetries induce a field dependent general coordinate transformation of the world volume space-time coordinates. From above, the general coordinate transformation for the world volume space-time coordinate differentials is given by

$$
d x^{\prime m}=d x^{n} G_{n}{ }^{m},
$$

where

$$
\begin{aligned}
G_{n}{ }^{m} & =\frac{\partial x^{\prime m}}{\partial x^{n}} \\
& =\delta_{n}{ }^{m}-i\left(\partial_{n} \theta \gamma^{0} \gamma^{m} \xi+\partial_{n} \lambda \gamma^{0} \gamma^{m} \eta\right)-\partial_{n} \phi b^{m}+\epsilon_{n}{ }^{m s} \alpha_{s} .
\end{aligned}
$$

The $G$-invariant interval can be formed by means of the metric tensor $g_{m n}$ so that $d s^{2}=d x^{m} g_{m n} d x^{n}=d s^{\prime 2}$ $=d x^{\prime m} g_{m n}^{\prime} d x^{\prime n}$ where the metric transforms as a tensor

$$
g_{m n}^{\prime}=G_{m}^{-1 r} g_{r s} G_{n}^{-1 s} .
$$

The metric can be constructed from the domain wall dreibein obtained from the Maurer-Cartan one-form.

\section{MAURER-CARTAN ONE-FORMS AND THE INVARIANT ACTION}

According to the coset construction method, the dreibein, the covariant derivatives of the Nambu-Goldstone fields and the spin connection can be obtained from the Maurer-Cartan one-forms. The Maurer-Cartan one-forms can be determined by use of the Feynman formula for the variation of an exponential operator along with the $\mathrm{B}-\mathrm{C}-\mathrm{H}$ formula $e^{A} B e^{-A}$ $=e^{\mathcal{L}_{A} \cdot B}$

$$
\begin{aligned}
e^{-i A} \delta e^{+i A}= & \int_{0}^{1} d t e^{-i t A}(i \delta A) e^{+i t A} \\
= & \frac{e^{\mathcal{L}-i A}-1}{\mathcal{L}_{-i A}} \cdot(i \delta A) \\
= & i \delta A-\frac{(-i)^{2}}{2}[A, \delta A]-\cdots \\
& -\frac{i^{(n+1)}}{(n+1) !} \underbrace{[A,[A, \ldots[A, \delta A] \cdots]}_{\mathrm{n}-\text { commutators }}-\cdots .
\end{aligned}
$$

The Maurer-Cartan one-forms are given as

$$
\begin{aligned}
\Omega^{-1} d \Omega= & i\left[\omega^{a} p_{a}+\bar{\omega}_{q i} q_{i}+\bar{\omega}_{s i} s_{i}+\omega_{Z} Z\right. \\
& \left.+\omega_{K}^{a} K_{a}+\omega_{M}^{a} M_{a}+\omega_{R} R\right]
\end{aligned}
$$

where the individual world volume one-forms are found to be

$$
\begin{aligned}
\omega^{a}= & \left(d x^{m}+i d \theta \gamma^{0} \gamma^{m} \theta+i d \lambda \gamma^{0} \gamma^{m} \lambda\right) \\
& \times\left(\delta_{m}{ }^{a}+\left(\cosh \sqrt{v^{2}}-1\right) \frac{v_{m} v^{a}}{v^{2}}\right) \\
& +\left(d \phi+d \theta \gamma^{0} \lambda-d \lambda \gamma^{0} \theta\right) \frac{\sinh \sqrt{v^{2}}}{\sqrt{v^{2}}} v^{a}
\end{aligned}
$$$$
\omega_{q i}=\cosh \frac{1}{2} \sqrt{v^{2}} d \theta_{i}-\frac{i}{2} \frac{\sinh \frac{1}{2} \sqrt{v^{2}}}{\frac{1}{2} \sqrt{v^{2}}}(\boldsymbol{} d \lambda)_{i}
$$

$$
\omega_{s i}=\cosh \frac{1}{2} \sqrt{v^{2}} d \lambda_{i}+\frac{i}{2} \frac{\sinh \frac{1}{2} \sqrt{v^{2}}}{\frac{1}{2} \sqrt{v^{2}}}(\boldsymbol{b} d \theta)_{i}
$$




$$
\begin{aligned}
\omega_{Z}= & \left(d \phi+d \theta \gamma^{0} \lambda-d \lambda \gamma^{0} \theta\right) \cosh \sqrt{v^{2}}+\left(d x^{m}\right. \\
& \left.+i d \theta \gamma^{0} \gamma^{m} \theta+i d \lambda \gamma^{0} \gamma^{m} \lambda\right) v_{m} \frac{\sinh \sqrt{v^{2}}}{\sqrt{v^{2}}} \\
\omega_{K}^{a}= & d v^{b}\left[\delta_{b}{ }^{a}+\left(\frac{\sinh \sqrt{v^{2}}}{\sqrt{v^{2}}}-1\right) P_{v T b}{ }^{a}\right] \\
\omega_{M}^{a}= & \left(\cosh \sqrt{v^{2}}-1\right) \frac{v_{b} d v_{c}}{v^{2}} \epsilon^{a b c} \\
\omega_{R}= & 0 .
\end{aligned}
$$

The Maurer-Cartan one-forms transform covariantly under all of the $G$ symmetries except the unbroken $D=3$ Lorentz transformation one-form $\omega_{M}^{a}$, which transforms with an additional shift $\left(h d h^{-1} \neq 0\right)$ under the broken $D=4$ Lorentz transformations, as required of a connection one-form. Explicitly, recalling that left multiplication by a group member induces a transformation in the world volume space-time coordinates and fields, $g \Omega=\Omega^{\prime} h$, the Maurer-Cartan oneforms transform as

$$
\left(\Omega^{-1} d \Omega\right)^{\prime}=h\left(\Omega^{-1} d \Omega\right) h^{-1}+h d h^{-1} .
$$

From this the dreibein, the covariant derivatives and the spin connection transformations can be obtained. In addition, as shown below, the $G$-covariant one-form $\omega_{Z}$ can be used to eliminate the would be Nambu-Goldstone field $v^{m}$ so that the independent degrees of freedom include only the NambuGoldstone modes for the spontaneously broken translation symmetry and supersymmetry. These correspond to excitations of the 2-brane into $N=1, D=4$ superspace directions "normal" to the spatial non-BPS domain wall brane. Towards this end, the world volume tangent space covariant coordinate basis differentials are given by the $\omega^{a}$ one-form. For a $G$-transformation they transform under the broken $D$ $=4$ Lorentz transformations and the unbroken $D=3$ Lorentz rotations according to their $D=3$ (local) Lorentz vector nature as given by $h$ in Eq. (2.10) (and are $R$ invariant). Writing $h$ as $h=e^{i \beta_{a}(g, v) M^{a}}$, the transformation of $\omega^{a}$ is given by

$$
\omega^{\prime a}=\omega^{b} L_{b}^{a},
$$

where the transformation is simply

$$
L_{b}{ }^{a}=\delta_{b}{ }^{a}+\beta^{c} \epsilon_{c b}{ }^{a}=\left(e^{\left.-i \beta_{c} \tilde{M}_{\text {vector }}^{c}\right)_{b}{ }^{a},}\right.
$$

with the $D=3$ Lorentz vector representation matrix $\left(\tilde{M}_{\text {vector } c}\right)_{a}{ }^{b}=i \epsilon_{c a}{ }^{b}$. The determinant of $L$ is unity: $\operatorname{det} L$ $=1$. (The remaining one-forms similarly transform according to their $D=3$ Lorentz character. Because of this local Lorentz structure group transformation property of the vector one-forms, their indices are denoted by letters from the beginning of the alphabet: $a, b, c, \ldots=0,1,2$.)

The two sets of coordinate basis differentials $d x^{m}$ and $\omega^{a}$ are related to each other through the dreibein $e_{m}{ }^{a}$

$$
\omega^{a}=d x^{m} e_{m}^{a}
$$

From Eq. (3.3) this yields

$$
\begin{aligned}
e_{m}{ }^{a}= & \left(\delta_{m}{ }^{b}+i \partial_{m} \theta \gamma^{0} \gamma^{b} \theta+\partial_{m} \lambda \gamma^{0} \gamma^{b} \lambda\right) \\
& \times\left(\delta_{b}{ }^{a}+\left(\cosh \sqrt{v^{2}}-1\right) \frac{v_{b} v^{a}}{v^{2}}+\left(\hat{\mathcal{D}}_{b} \phi+\hat{\mathcal{D}}_{b} \theta \gamma^{0} \lambda\right.\right. \\
& \left.\left.-\theta \gamma^{0} \hat{\mathcal{D}}_{b} \lambda\right) v^{a} \frac{\sinh \sqrt{v^{2}}}{\sqrt{v^{2}}}\right)
\end{aligned}
$$

with the Akulov-Volkov derivative $\hat{\mathcal{D}}_{a}=\hat{e}_{a}^{-1 m} \partial_{m}$ defined below [see Eq. (4.13)]. Under a $G$-transformation the dreibein transforms with one world index and one tangent space (structure group) index as

$$
e_{m}^{\prime a}=G_{m}^{-1 n} e_{n}^{b} L_{b}^{a}
$$

and likewise for the inverse dreibein $e_{a}^{\prime-1 m}$ $=L_{a}^{-1 b} e_{b}^{-1 n} G_{n}{ }^{m}$. By direct calculation from the form of $L$, Eq. (3.6), the flat tangent space metric, $\eta_{a b}$, is invariant

$$
\eta_{a b}^{\prime}=L_{a}^{c} \eta_{c d} L_{b}^{d}=\eta_{a b}
$$

The metric tensor is given in terms of the dreibein as

$$
g_{m n}=e_{m}{ }^{a} \eta_{a b} e_{n}{ }^{b}
$$

the transformation properties of which are given by Eq. (2.15) and follow from those of the dreibein and the flat tangent space metric. Consequently the covariant MaurerCartan one-form can be used to express the invariant interval as

$$
d s^{2}=d x^{m} g_{m n} d x^{n}=\omega^{a} \eta_{a b} \omega^{b} .
$$

The leading term in the $D=4$ super-Poincare invariant action is given by the "cosmological constant" term

$$
\Gamma=-\sigma \int d^{3} x \operatorname{det} e
$$

with the brane tension parameter $\sigma$. The Lagrangian is the constant brane tension integrated over the area of the brane. The action is invariant

$$
\begin{aligned}
\Gamma^{\prime} & =-\sigma \int d^{3} x^{\prime} \operatorname{det} e^{\prime}=-\sigma \int\left(d^{3} x \operatorname{det} G\right)\left(\operatorname{det} G^{-1} \operatorname{det} e \operatorname{det} L\right) \\
& =-\sigma \int d^{3} x \operatorname{det} e=\Gamma
\end{aligned}
$$

\section{DREIBEINE, COVARIANT DERIVATIVES AND BRANE DYNAMICS}

The world volume exterior derivative, $d=d x^{m} \partial_{m}$, can also be written in terms of the fully $G$-covariant one-form basis

$$
d=d x^{m} \partial_{m}=\omega^{a} e_{a}^{-1 m} \partial_{m} \equiv \omega^{a} \mathcal{D}_{a}
$$


with the fully $G$-covariant derivative

$$
\mathcal{D}_{a} \equiv e_{a}^{-1 m} \partial_{m} .
$$

The exterior derivative is fully $G$-invariant $d^{\prime}=d$ since the coordinate derivative transforms inversely to the coordinate differential: $\partial_{m}^{\prime}=G_{m}^{-1 n} \partial_{n}$. Hence, $\mathcal{D}_{a}$ transforms as $\mathcal{D}_{a}^{\prime}$ $=L_{a}^{-1 b} \mathcal{D}_{b}$.

For each one-form, $\omega_{Q_{\varphi}}$, with $Q_{\varphi}=\left\{q_{i}, s_{i}, Z, K^{m}\right\}$, the respective covariant derivative of the related NambuGoldstone field, $\varphi=\left\{\theta_{i}, \lambda_{i}, \phi, v^{m}\right\}$, is defined according to

$$
\begin{aligned}
\omega_{Q_{\varphi}} & \equiv \omega^{a} \nabla_{a} \varphi=d x^{m} e_{m}{ }^{a} \nabla_{a} \varphi \\
& \equiv d x^{m} \omega_{Q_{\varphi} m}=\omega^{a} e_{a}^{-1 m} \omega_{Q_{\varphi} m} .
\end{aligned}
$$

Hence it is obtained that

$$
\nabla_{a} \varphi=e_{a}^{-1 m} \omega_{Q_{\varphi} m}
$$

or the inverse

$$
e_{m}{ }^{a} \nabla_{a} \varphi=\omega_{Q_{\varphi} m} .
$$

Recall that each one-form begins with the space-time derivative of the associated Nambu-Goldstone field: $\omega_{Q_{\varphi} m}=\partial_{m} \varphi$ $+\cdots$.

Besides the fully $G$-covariant basis of one-forms, partially covariant bases associated with restricted motions in the coset manifold can be defined. In particular the basis obtained from motion in the manifold with the coset coordinate $v^{n}=0$ is a $D=3$ Lorentz but not $D=4$ Lorentz covariant one-form basis. Most directly these one-forms, dreibein and partially covariant derivatives can be obtained by taking the $v^{n}$ field to zero in the above expressions, for example, $\hat{\omega}^{a}$ $\left.\equiv \omega^{a}\right|_{v^{n}=0}$. Alternatively, since the Maurer-Cartan one-forms can be built-up sequentially by including the different symmetry generators

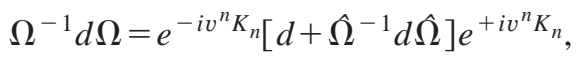

where the $\hat{\Omega}$ includes the remaining generators, the partially covariant one-forms are given by

$$
\begin{aligned}
\hat{\Omega}^{-1} d \hat{\Omega} \equiv & i\left[\hat{\omega}^{a} p_{a}+\overline{\hat{\omega}}_{q i} q_{i}+\overline{\hat{\omega}}_{s i} s_{i}+\hat{\omega}_{Z} Z\right] \\
= & i\left[d x^{a}+i d \theta \gamma^{0} \gamma^{a} \theta+i d \lambda \gamma^{0} \gamma^{a} \lambda\right] p_{a} \\
& +i d \bar{\theta} q+i d \overline{\lambda s}+i\left[d \phi+d \theta \gamma^{0} \lambda\right. \\
& \left.-d \lambda \gamma^{0} \theta\right] Z .
\end{aligned}
$$

The space-time coordinate differentials can be expressed in terms of this one-form basis through the Akulov-Volkov dreibein

$$
\hat{\omega}^{a}=d x^{m} \hat{e}_{m}^{a} .
$$

From Eqs. (4.7) and (4.8) [or $\hat{e}_{m}{ }^{a}=\left.e_{m}{ }^{a}\right|_{v^{n}=0}$ in Eq. (3.8)]

$$
\hat{e}_{m}{ }^{a}=A_{m}{ }^{a}
$$

where the Akulov-Volkov matrix $A_{m}{ }^{a}$ is defined as

$$
A_{m}{ }^{a}=\delta_{m}{ }^{a}+i \partial_{m} \theta \gamma^{0} \gamma^{a} \theta+i \partial_{m} \lambda \gamma^{0} \gamma^{a} \lambda .
$$

From these the $D=3$ SUSY $\mathrm{SO}(1,2)$ covariant derivatives follow

$$
d=d x^{m} \partial_{m} \equiv \hat{\omega}^{a} \hat{\mathcal{D}}_{a},
$$

whence $\hat{\mathcal{D}}_{a}=\hat{e}_{a}^{-1 m} \partial_{m}$ where the inverse dreibein $\hat{e}_{a}^{-1 m}$, so that $\hat{e}_{a}^{-1{ }^{m}} \hat{e}_{m}{ }^{b}=\delta_{a}{ }^{b}$ and $\hat{e}_{m}{ }^{a} \hat{e}_{a}^{-1 n}=\delta_{m}{ }^{n}$, is given by

$$
\hat{e}_{a}^{-1 m}=A_{a}^{-1 m} .
$$

Hence the partial covariant Akulov-Volkov derivative is obtained

$$
\hat{\mathcal{D}}_{a}=\hat{e}_{a}^{-1}{ }^{m} \partial_{m}=A_{a}^{-1}{ }^{m} \partial_{m} .
$$

The $G$-transformation properties of the partially covariant one-forms, Eq. (4.7), can be found from the factorization of the coset element and the general transformation law. Writing $\Omega=\hat{\Omega} \Omega_{K}$, the transformation law $g \Omega=\Omega^{\prime} h$ implies that

$$
\left(\hat{\Omega}^{-1} d \hat{\Omega}\right)^{\prime}=\hat{h}\left(\hat{\Omega}^{-1} d \hat{\Omega}\right) \hat{h}^{-1},
$$

where now $\hat{h}$ involves the broken and unbroken Lorentz generators but with the field independent transformation parameters of $g, \hat{h}=e^{i \rho R} e^{i b^{n} K_{n}} e^{i \alpha^{n} M_{n}}$. In particular, this yields the noncovariant transformation law for $\hat{\omega}^{a}$ (even so, the use of indices from the beginning of the alphabet is retained)

$$
\begin{aligned}
\hat{\omega}^{\prime a} & =\hat{\omega}^{b} \hat{L}_{b}{ }^{a} \\
& =\hat{\omega}^{b}\left(\delta_{b}{ }^{a}+\alpha^{c} \epsilon_{c b}{ }^{a}-\hat{\nabla}_{b} \phi b^{a}\right),
\end{aligned}
$$

with the partially covariant derivative of $\phi, \hat{\nabla}_{a} \phi$, given by the Maurer-Cartan one-form $\hat{\omega}_{Z}$,

$$
\hat{\omega}_{Z}=\hat{\omega}^{a} \hat{\nabla}_{a} \phi=\hat{\omega}^{a}\left[\hat{\mathcal{D}}_{a} \phi+\hat{\mathcal{D}}_{a} \theta \gamma^{0} \lambda-\theta \gamma^{0} \hat{\mathcal{D}}_{a} \lambda\right] .
$$

Hence, the Akulov-Volkov derivative transforms as

$$
\hat{\mathcal{D}}_{a}^{\prime}=\hat{L}_{a}^{-1 b} \hat{\mathcal{D}}_{b},
$$

and as such is not fully $G$-covariant due to its variation under the broken Lorentz transformations $\left(b^{n} \neq 0\right)$. As with $\hat{\omega}^{a}$, the Akulov-Volkov derivative $\hat{\mathcal{D}}_{a}$ is only $S O(1,2)$ partially covariant.

It is useful to expand the one-forms of the fully $S O(1,3)$ covariant basis in terms of the $S O(1,2)$ covariant basis. The two bases are related as 


$$
\begin{aligned}
\hat{\omega}^{a} & =\omega^{b} e_{b}^{-1}{ }^{m} \hat{e}_{m}{ }^{a} \\
\omega^{a} & =\hat{\omega}^{b} \hat{e}_{b}^{-1 m} e_{m}{ }^{a}
\end{aligned}
$$

which follows from the superspace coordinate differentials

$$
d x^{m}=\omega^{a} e_{a}^{-1 m}=\hat{\omega}^{a} \hat{e}_{a}^{-1 m} .
$$

Likewise, through the exterior derivative, $d=d x^{m} \partial_{m}$ $=\omega^{a} \mathcal{D}_{a}=\hat{\omega}^{a} \hat{\mathcal{D}}_{a}$, the (partially)covariant derivatives are related

$$
\begin{aligned}
& \hat{\mathcal{D}}_{a}=\hat{e}_{a}^{-1}{ }^{m} e_{m}{ }^{b} \mathcal{D}_{b} \\
& \mathcal{D}_{a}=e_{a}^{-1}{ }^{m} \hat{e}_{m}{ }^{b} \hat{\mathcal{D}}_{b} .
\end{aligned}
$$

In particular the $G$-covariant coordinate differential oneform, $\omega^{a}$, has a simple relation to the partially covariant coordinate differential one-form, $\hat{\omega}^{a}$,

$$
\omega^{a}=\hat{\omega}^{b} N_{b}^{a},
$$

where the Nambu-Goto dreibein, $N_{b}{ }^{a}$, is found from Eqs. (3.3) and (4.7)

$$
\begin{aligned}
N_{b}{ }^{a}= & \delta_{b}{ }^{a}+\left(\cosh \sqrt{v^{2}}-1\right) \frac{v_{b} v^{a}}{v^{2}}+\left(\hat{\mathcal{D}}_{b} \phi+\hat{\mathcal{D}}_{b} \theta \gamma^{0} \lambda\right. \\
& \left.-\theta \gamma^{0} \hat{\mathcal{D}}_{b} \lambda\right) v^{a} \frac{\sinh \sqrt{v^{2}}}{\sqrt{v^{2}}} .
\end{aligned}
$$

Similarly the dreibeine are related, Eq. (3.8),

$$
e_{m}^{a}=\hat{e}_{m}^{b} N_{b}^{a} .
$$

Thus the invariant action takes on a factorized form

$$
\Gamma=-\sigma \int d^{3} x \operatorname{det} e=-\sigma \int d^{3} x \operatorname{det} \hat{e} \operatorname{det} N
$$

The dete has the usual form of the Akulov-Volkov determinant for spontaneously broken $N=2, D=3$ supersymmetry. The $\operatorname{det} N$ term can be evaluated to yield the SUSY generalization to the Nambu-Goto action for the $p=2$ brane allowing for its motion into the Grassmann directions of the target $N=1, D=4$ superspace

$$
\begin{aligned}
\operatorname{det} N= & \cosh \sqrt{v^{2}}\left[1+\left(\hat{\mathcal{D}}_{a} \phi+\hat{\mathcal{D}}_{a} \theta \gamma^{0} \lambda\right.\right. \\
& \left.\left.-\theta \gamma^{0} \hat{\mathcal{D}}_{a} \lambda\right) v^{a} \frac{\tanh \sqrt{v^{2}}}{\sqrt{v^{2}}}\right] .
\end{aligned}
$$

There are two equivalent ways in which to proceed in order to simplify the action by the elimination of the $v^{m}$ field. The Euler-Lagrange approach is a result of the fact that the action depends only on $v^{m}$ and not its derivatives. Hence the $v^{m}$ field equation, $\delta \Gamma / \delta v^{m}=0$, will express $v^{m}$ in terms of the independent Nambu-Goldstone fields, $\phi, \theta$ and $\lambda$. Alternatively, the Maurer-Cartan one-form associated with the broken translation generator $Z$ can be $G$-covariantly set to zero. Expanding the $\omega_{Z}$ one-form in terms of the $\hat{\omega}^{a}$ basis gives

$$
\begin{aligned}
\omega_{Z}= & \hat{\omega}^{a} \cosh \sqrt{v^{2}}\left[\left(\hat{\mathcal{D}}_{a} \phi+\hat{\mathcal{D}}_{a} \theta \gamma^{0} \lambda-\theta \gamma^{0} \hat{\mathcal{D}}_{a} \lambda\right)\right. \\
& \left.+v_{a} \frac{\tanh \sqrt{v^{2}}}{\sqrt{v^{2}}}\right] .
\end{aligned}
$$

Setting this to zero results in the "inverse Higgs mechanism"

$$
v_{a} \frac{\tanh \sqrt{v^{2}}}{\sqrt{v^{2}}}=-\left(\hat{\mathcal{D}}_{a} \phi+\hat{\mathcal{D}}_{a} \theta \gamma^{0} \lambda-\theta \gamma^{0} \hat{\mathcal{D}}_{a} \lambda\right)=-\hat{\nabla}_{a} \phi
$$

This result is also obtained in the Euler-Lagrange approach. Substituting this into the determinant of the Nambu-Goto dreibein yields the SUSY generalization of the Nambu-Goto Lagrangian as given in Eq. (1.35)

$$
\begin{aligned}
\operatorname{det} N & =\frac{1}{\cosh \sqrt{v^{2}}} \\
& =\sqrt{1-\left(\hat{\mathcal{D}}_{a} \phi+\hat{\mathcal{D}}_{a} \theta \gamma^{0} \lambda-\theta \gamma^{0} \hat{\mathcal{D}}_{a} \lambda\right)^{2}} .
\end{aligned}
$$

Hence the complete $G$-invariant Nambu-Goto-AkulovVolkov action is given by

$$
\begin{aligned}
\Gamma= & -\sigma \int d^{3} x\left\{\operatorname{det}\left[\delta_{m}{ }^{a}+i \partial_{m} \theta \gamma^{0} \gamma^{a} \theta+i \partial_{m} \lambda \gamma^{0} \gamma^{a} \lambda\right]\right. \\
& \left.\times \sqrt{1-\left(\hat{\mathcal{D}}_{b} \phi+\hat{\mathcal{D}}_{b} \theta \gamma^{0} \lambda-\theta \gamma^{0} \hat{\mathcal{D}}_{b} \lambda\right)^{2}}\right\}
\end{aligned}
$$

Returning to Eq. (4.25)and treating all fields as independent leads to the $\phi$ equation of motion as the $D=3$ Bianchi identity for the field strength vector $F^{m}$

$$
0=\frac{\delta \Gamma}{\delta \phi}=\partial_{m} F^{m}
$$

where

$$
F^{m}=\operatorname{det} \hat{e} v^{a} \hat{e}_{a}^{-1 m} \frac{\sinh \sqrt{v^{2}}}{\sqrt{v^{2}}} .
$$

Substituting this back into the Lagrangian yields

$$
\operatorname{det} \hat{e} \operatorname{det} N=\operatorname{det} \hat{e} \cosh \sqrt{v^{2}}+F^{m}\left[\partial_{m} \phi+\partial_{m} \theta \gamma^{0} \lambda-\theta \gamma^{0} \partial_{m} \lambda\right] \text {. }
$$

Exploiting the definition of $F^{m}$ so that

$$
\frac{v^{a} v^{b}}{v^{2}}=\frac{\left(F^{m} \hat{e}_{m}{ }^{a}\right)\left(F^{n} \hat{e}_{n}{ }^{b}\right)}{(F \hat{e})^{2}}
$$

results in 


$$
\cosh \sqrt{v^{2}}=\sqrt{\left(1+\frac{(F \hat{e})^{2}}{(\operatorname{det} \hat{e})^{2}}\right)}
$$

Integrating this over the world volume, the non-BPS $p=2$ brane supersymmetric Nambu-Goto-Akulov-Volkov action is dual to the D2-brane supersymmetric Born-Infeld action

$$
\begin{aligned}
\Gamma & =-\sigma \int d^{3} x\left(\sqrt{\operatorname{det}\left(\hat{g}_{m n}+F_{m n}\right)}+F^{n}\left[\partial_{n} \theta \gamma^{0} \lambda-\theta \gamma^{0} \partial_{n} \lambda\right]\right) \\
& =-\sigma \int d^{3} x\left(\sqrt{\operatorname{det} \hat{g}+F^{m} \hat{g}_{m n} F^{n}}+F^{n}\left[\partial_{n} \theta \gamma^{0} \lambda-\theta \gamma^{0} \partial_{n} \lambda\right]\right),
\end{aligned}
$$

where the Akulov-Volkov metric is given by $\hat{g}_{m n}$ $=\hat{e}_{m}{ }^{a} \eta_{a b} \hat{e}_{n}{ }^{b}$ and $F_{m n}=\epsilon_{m n r} F^{r}$.

\section{BRANE LOCALIZED MATTER FIELDS}

The matter fields localized on the brane are characterized by their $D=3$ Lorentz transformation properties. A scalar field, $S(x)$, is in the trivial representation of the Lorentz group: $M^{a} \rightarrow\left(\tilde{M}^{a}\right)=0$. Fermion fields, $\psi_{i}(x)$, are in the spinor representation: $M^{a} \rightarrow\left(\tilde{M}^{a}\right)_{i j}=-1 / 2 \gamma_{i j}^{a}$. Each matter field, $M(x)$, transforms under $G$ as

$$
M^{\prime}\left(x^{\prime}\right) \equiv \widetilde{h} M(x)
$$

where $\tilde{h}$ is given by $h$, Eq. (2.10), with $M^{a}$ replaced by $\tilde{M}^{a}$ and the field's $R$-weight phase, $\rho_{M}$, a model dependent convention

$$
\tilde{h}=e^{i \beta_{a}(g, v) \tilde{M}^{a}} e^{i \rho_{M}} .
$$

The covariant derivative for the matter field is defined using the spin connection one-form

$$
\nabla M \equiv\left(d+i \omega_{M}^{a} \tilde{M}_{a}\right) M
$$

The transformation properties of the covariant derivative,

$$
(\nabla M)^{\prime}\left(x^{\prime}\right)=\tilde{h} \nabla M(x),
$$

are obtained from the invariant nature of the exterior derivative $d$, the field dependent transformation equation for $M$ and the inhomogeneous transformation property of the connection. For infinitesimal $G$ transformations recall that $h d h^{-1}$ $=-i d \beta^{a} M_{a}$ so that the connection one-form transforms according to [with $\left(\tilde{M}_{\text {vector }}^{a}\right)_{b c}=i \epsilon_{b c}^{a}$ ]

$$
\begin{aligned}
\omega_{M}^{\prime a} & =\omega_{M}^{b} L_{b}^{a}-d \beta^{a} \\
& =\omega_{M}{ }^{b}\left(e^{\left.-i \beta_{c} \tilde{M}_{\text {vector }}^{c}\right)_{b}{ }^{a}-d \beta^{a} .}\right.
\end{aligned}
$$

The covariant derivative transformation law equation (5.4) follows.
Expanding the covariant derivative one-form in terms of the tangent space covariant coordinate basis differentials, $\omega^{a}$, the component form of the covariant derivative is obtained

$$
\nabla_{a} M=\left(\mathcal{D}_{a}+i \Gamma_{a}{ }^{b} \tilde{M}_{b}\right) M
$$

where $\Gamma_{a}{ }^{b}$ are the components of the connection, $\omega_{M}{ }^{b}$ $=\omega^{a} \Gamma_{a}{ }^{b}$. Also, in component form, the connection transformation law is found to be

$$
\Gamma_{a}^{\prime b}=L_{a}^{-1 c} \Gamma_{c}^{d} L_{d}^{b}-L_{a}^{-1 c} \mathcal{D}_{c} \beta^{b} .
$$

Since the covariant coordinate differentials transform according to the $D=3$ (field dependent)local Lorentz (structure) group vector representation matrices, $L_{a}{ }^{b}$, Eq. (3.5), the component form of the covariant derivative has the $G$ transformation law

$$
\left(\nabla_{a} M\right)^{\prime}\left(x^{\prime}\right)=\tilde{h} L_{a}^{-1 b} \nabla_{b} M(x)
$$

For scalar matter fields the covariant derivative is simply the covariant space-time derivative $\mathcal{D}_{a}=e_{a}^{-1 m} \partial_{m}$ :

$$
\nabla_{a} S(x)=\mathcal{D}_{a} S(x) .
$$

Since $S$ is invariant, $S^{\prime}\left(x^{\prime}\right)=S(x)$, the covariant derivative transforms as a tangent space vector

$$
\left(\nabla_{a} S\right)^{\prime}\left(x^{\prime}\right)=L_{a}^{-1 b} \nabla_{b} S(x) .
$$

Because the flat tangent space metric, $\eta^{a b}$, is invariant, the leading terms in the brane width expansion of the $G$-invariant action for the scalar matter field are obtained as

$$
\Gamma_{S}=\int d^{3} x \operatorname{det} e \mathcal{L}_{S}
$$

with the scalar field invariant Lagrangian (that is invariant under total $G$ transformations and hence a scalar density under intrinsic $G$ transformations) given by

$$
\mathcal{L}_{S}=\left(\nabla_{a} S \eta^{a b} \nabla_{b} S\right)-V(S)
$$

where the scalar field potential $V(S)$ is an arbitrary function of $S$.

The fermion matter field $\psi_{i}(x)$ transforms as the $D=3$ Lorentz group spinor representation

$$
\psi_{i}^{\prime}\left(x^{\prime}\right)=\tilde{h}_{i j} \psi_{j}(x),
$$

with (suppressing the $R$-transformation weight)

$$
\tilde{h}_{i j}=\left(e^{-(i / 2) \beta_{a} \gamma^{a}}\right)_{i j}
$$

Hence the bilinear product, $\bar{\psi} \psi$, is invariant, $(\bar{\psi} \psi)^{\prime}\left(x^{\prime}\right)$ $=(\bar{\psi} \psi)(x)$. The vector bilinear product transforms as a tangent space vector, $\left(\bar{\psi} \gamma^{a} \psi\right)^{\prime}\left(x^{\prime}\right)=\left(\bar{\psi} \gamma^{b} \psi\right)(x) L_{b}{ }^{a}$. The covariant derivative now involves the spin connection and, in component form, is given by 


$$
\nabla_{a} \psi_{i}=\mathcal{D}_{a} \psi_{i}-\frac{i}{2} \Gamma_{a}^{b} \gamma_{b i j} \psi_{j}
$$

The fermion covariant derivative transforms according to

$$
\left(\nabla_{a} \psi_{i}\right)^{\prime}\left(x^{\prime}\right)=\widetilde{h}_{i j} L_{a}^{-1 b} \nabla_{b} \psi_{j}
$$

The invariant kinetic energy bilinear is given by $\left(\bar{\psi} \gamma^{a} \nabla_{a} \psi\right)$ so that

$$
\left(\bar{\psi} \gamma^{a} \nabla_{a} \psi\right)^{\prime}\left(x^{\prime}\right)=\left(\bar{\psi} \gamma^{a} \nabla_{a} \psi\right)(x) .
$$

The $G$ invariant action has the form

$$
\Gamma_{f}=\int d^{3} x \operatorname{det} e \mathcal{L}_{f},
$$

where the invariant Lagrangian involves the fermion and scalar matter fields

$$
\mathcal{L}_{f}=i \bar{\psi} \gamma^{a} \nabla_{a} \psi-m \bar{\psi} \psi+Y(S, \bar{\psi} \psi),
$$

with the generalized Yukawa coupling to the scalar fields, $Y(S, \bar{\psi} \psi)$. In the case of a single species of $D=3$ Majorana fermion, the Yukawa term terminates at the form $y(S) \bar{\psi} \psi$, with the arbitrary Yukawa coupling function $y(S)$.

The above covariant derivatives were expanded in the fully covariant $\omega^{a}$ basis, the relation to the expansion in terms of the partially covariant $\hat{\omega}^{a}$ basis can also be obtained. As found above, the scalar and fermion covariant derivatives in the fully $G$ covariant basis are

$$
\begin{aligned}
\nabla_{a} S & =\mathcal{D}_{a} S \\
\nabla_{a} \psi_{i} & =\mathcal{D}_{a} \psi_{i}-\frac{i}{2} \Gamma_{a}{ }^{b} \gamma_{b i j} \psi_{j} .
\end{aligned}
$$

The covariant derivatives are related through the exterior derivative and the dreibeine as in Eqs. (4.18)-(4.20). The coordinate differentials are related according to

$$
\begin{aligned}
& \omega^{a}=d x^{m} e_{m}{ }^{a} \quad d x^{m}=\omega^{a} e_{a}^{-1 m} \\
& \hat{\omega}^{a}=d x^{m} \hat{e}_{m}^{a} \quad d x^{m}=\hat{\omega}^{a} \hat{e}_{a}^{-1 m} \\
& \omega^{a}=\hat{\omega}^{b} N_{b}{ }^{a} \quad e_{a}^{-1 n} \hat{e}_{n}{ }^{b}=N_{a}^{-1 b}
\end{aligned}
$$

The relation between the covariant derivatives is found through the exterior derivative

$$
\begin{aligned}
d & =d x^{m} \partial_{m}=\omega^{a} \mathcal{D}_{a}=\hat{\omega}^{a} \hat{\mathcal{D}}_{a} \\
& =d x^{m} e_{m}{ }^{a} \mathcal{D}_{a}=d x^{m} \hat{e}_{m}{ }^{a} \hat{\mathcal{D}}_{a},
\end{aligned}
$$

with, as previously defined,

$$
\begin{aligned}
& \mathcal{D}_{a}=e_{a}^{-1 m} \partial_{m} \\
& \hat{\mathcal{D}}_{a}=\hat{e}_{a}^{-1 m} \partial_{m} .
\end{aligned}
$$

The relation between (partially) covariant derivatives is secured [see Eq. (4.20))

$$
\begin{aligned}
\mathcal{D}_{a} & =e_{a}^{-1 m} \hat{e}_{m}^{b} \hat{\mathcal{D}}_{b} \\
& =N_{a}^{-1 b} \hat{\mathcal{D}}_{b} .
\end{aligned}
$$

Recall that $\hat{\mathcal{D}}_{a}=A_{a}^{-1 m} \partial_{m}$ is just the SUSY covariant AkulovVolkov derivative, Eq. (4.13).

Besides the derivatives, also the connection can be expressed in terms of the partially covariant coordinate differentials. Recall the connection from Eq. (3.3)

$$
\begin{aligned}
\omega_{M}^{b} & =\omega^{a} \Gamma_{a}^{b}=\hat{\omega}^{a} \hat{\Gamma}_{a}^{b} \\
& =\left(\cosh \sqrt{v^{2}}-1\right) \epsilon^{b a c} \frac{v_{a} d v_{c}}{v^{2}} .
\end{aligned}
$$

Upon application of the $\omega_{Z}=0$ constraint, the above expression becomes

$$
\omega_{M}^{b}=(1 / \operatorname{det} N-1) \epsilon^{b a c} \frac{\varphi_{a} d \varphi_{c}}{\varphi^{2}},
$$

where $\varphi_{a}$ is defined by

$$
\varphi_{a}=\hat{\mathcal{D}}_{a} \phi+\hat{\mathcal{D}}_{a} \theta \gamma^{0} \lambda-\theta \gamma^{0} \hat{\mathcal{D}}_{a} \lambda .
$$

In components in the fully covariant basis, the connection involves the fully covariant derivative

$$
\Gamma_{a}{ }^{b}=\left(\cosh \sqrt{v^{2}}-1\right) \epsilon^{b c d} \frac{v_{c} \mathcal{D}_{a} v_{d}}{v^{2}} .
$$

Using the relation between the two bases, the connection components are related according to

$$
\Gamma_{a}^{b}=N_{a}^{-1 c} \hat{\Gamma}_{c}^{b},
$$

with

$$
\hat{\Gamma}_{a}^{b}=\left(\cosh \sqrt{v^{2}}-1\right) \epsilon^{b c d} \frac{v_{c} \hat{\mathcal{D}}_{a} v_{d}}{v^{2}},
$$

which, after imposition of the $\omega_{Z}=0$ constraint, becomes

$$
\hat{\Gamma}_{a}^{b}=(1 / \operatorname{det} N-1) \epsilon^{b c d} \frac{\varphi_{c} \hat{\mathcal{D}}_{a} \varphi_{d}}{\varphi^{2}} .
$$

The matter field covariant derivatives then have the form

$$
\begin{aligned}
\nabla_{a} S & =\mathcal{D}_{a} S=N_{a}^{-1 b} \hat{\nabla}_{b} S \\
\nabla_{a} \psi_{i} & =N_{a}^{-1 b} \hat{\nabla}_{b} \psi_{i},
\end{aligned}
$$

with the scalar and fermion fields' partially covariant derivatives defined as 


$$
\begin{aligned}
\hat{\nabla}_{a} S & =\hat{\mathcal{D}}_{a} S \\
\hat{\nabla}_{a} \psi_{i} & =\hat{\mathcal{D}}_{a} \psi_{i}-\frac{i}{2} \hat{\Gamma}_{a}^{b} \gamma_{b i j} \psi_{j} .
\end{aligned}
$$

The matter field action can be written in terms of the partially covariant derivatives but the Nambu-Goto dreibein and metric are needed in order to restore full $G$ invariance. Using the Nambu-Goto dreibein $N$, its inverse is

$$
N_{a}^{-1 b}=\delta_{a}^{b}-\frac{1}{\cosh \sqrt{v^{2}}+d_{c} v^{c}}\left(N_{a}^{b}-\delta_{a}^{b}\right)
$$

where

$$
d_{a}=\varphi_{a} \frac{\sinh \sqrt{v^{2}}}{\sqrt{v^{2}}}
$$

and $N$ is given in Eq. (4.22)

$$
N_{a}{ }^{b}=P_{v T a}{ }^{b}+\cosh \sqrt{v^{2}} P_{v L a}{ }^{b}+d_{a} v^{b} .
$$

Upon application of the $\omega_{Z}=0 G$-covariant constraint, these dreibeine reduce to

$$
\begin{gathered}
N_{a}{ }^{b}=\delta_{a}{ }^{b}+\frac{1-\cosh \sqrt{v^{2}}}{\cosh \sqrt{v^{2}}} \frac{v_{a} v^{b}}{v^{2}} \\
N_{a}^{-1 b}=\delta_{a}{ }^{b}+\left(\cosh \sqrt{v^{2}}-1\right) P_{v L a}^{b} .
\end{gathered}
$$

This can be used to form the Nambu-Goto metric

$$
\begin{aligned}
n^{a b} & =N_{c}^{-1 a} \eta^{c d} N_{d}^{-1 b} \\
& =\eta^{a b}+\frac{v^{a} v^{b}}{v^{2}} \sinh ^{2} \sqrt{v^{2}} .
\end{aligned}
$$

The invariant interval can be written as

$$
d s^{2}=d x^{m} g_{m n} d x^{n}=\omega^{a} \eta_{a b} \omega^{b}=\hat{\omega}^{a} n_{a b} \hat{\omega}^{b},
$$

with $n_{a b}=N_{a}{ }^{c} \eta_{c d} N_{b}{ }^{d}=\eta_{a b}-\left(v_{a} v_{b} / v^{2}\right) \tanh ^{2} \sqrt{v^{2}}$.

The fully $G$-invariant kinetic energy term for the scalar field in terms of the partially covariant derivatives then becomes

$$
\nabla_{a} S \eta^{a b} \nabla_{b} S=\hat{\nabla}_{a} S n^{a b} \hat{\nabla}_{b} S
$$

Likewise, the fully $G$-invariant fermion kinetic energy becomes

$$
i \bar{\psi} \gamma^{a} \nabla_{a} \psi=i \bar{\psi} \hat{\gamma}^{a} \hat{\nabla}_{a} \psi
$$

where the Dirac matrices in the partially covariant basis are defined by means of the Nambu-Goto dreibein

$$
\hat{\gamma}^{a}=\gamma^{b} N_{b}^{-1 a} .
$$

The fully $G$-invariant matter action then takes the form

$$
\Gamma_{\text {matter }}=\Gamma_{S}+\Gamma_{f}=\int d^{3} x \operatorname{det} e \mathcal{L}_{\text {matter }},
$$

where the invariant matter Lagrangian can be written as

$$
\begin{aligned}
\mathcal{L}_{\text {matter }} & =\mathcal{L}_{S}+\mathcal{L}_{f} \\
& =\hat{\nabla}_{a} S n^{a b} \hat{\nabla}_{b} S-V(S)+i \bar{\psi} \hat{\gamma}^{a} \hat{\nabla}_{a} \psi-m \bar{\psi} \psi+Y(S, \bar{\psi} \psi) .
\end{aligned}
$$

\section{ACKNOWLEDGMENTS}

M.N. would like to thank Koji Hashimoto and T.t.V. would like to thank Mikhail Shifman, Arkady Vainshtein and Mikhail Voloshin for interesting discussions. The work of T.E.C. and M.N. was supported in part by the U.S. Department of Energy under grant DE-FG02-91ER40681 (Task B).

\section{APPENDIX: CONVENTIONS}

The $D=3$ Dirac matrix conventions are (labeling all space-time indices by $m, n, \ldots=0,1,2$ for convenience here)

$$
\begin{aligned}
\eta_{m n} & =(+,-,-), \quad \epsilon^{012}=+1 \\
\epsilon_{m n l} \epsilon^{l r s} & =\delta_{m}^{r} \delta_{n}^{s}-\delta_{m}^{s} \delta_{n}^{r} \\
\epsilon_{m n l} \epsilon^{n l s} & =+2 \delta_{m}^{s} \\
\gamma^{m}=\left(\sigma^{2}, i \sigma^{1}, i \sigma^{3}\right) & =-\gamma^{m \star}, \quad \sigma^{1} \sigma^{2}=i \sigma^{3} \\
C & =\gamma^{0}=\sigma^{2}=C^{-1}, \quad C^{T}=-C \\
\gamma^{m} \gamma^{n} & =\eta^{m n} 1+i \epsilon^{m n r} \gamma_{r} \\
\gamma^{m} \gamma^{n} \gamma^{r} & =+i \epsilon^{m n r} 1+\eta^{m n} \gamma^{r}-\eta^{m r} \gamma^{n}+\eta^{n r} \gamma^{m} \\
\left\{\gamma^{m}, \gamma^{n}\right\} & =+2 \eta^{m n} \\
\gamma^{m n} & \equiv \frac{i}{2}\left[\gamma^{m}, \gamma^{n}\right]=-\epsilon^{m n r} \gamma_{r} \\
\gamma^{m T} & =-\gamma^{0} \gamma^{m} \gamma^{0}=-\gamma^{m \dagger}, \quad\left(\gamma^{0} \gamma^{m}\right)^{T}=\gamma^{0} \gamma^{m} \\
\gamma_{i j}^{m} \gamma_{j i}^{n} & =2 \eta^{m n} \\
\gamma_{i j}^{k} \gamma_{j k}^{l} \gamma_{k i}^{m} & =2 i \epsilon^{k l m} \\
\gamma^{12} & =-\sigma^{2}=-\gamma^{21} \\
\gamma^{01} & =i \sigma^{3}=-\gamma^{10} \\
\gamma^{02} & =-i \sigma^{1}=-\gamma^{20} \\
\gamma^{m} C & =\left(1,-\sigma^{3}, \sigma^{1}\right)
\end{aligned}
$$




$$
\left(\gamma^{m} C\right)_{i j} A_{m}=\left(\begin{array}{cc}
\left(A_{0}-A_{1}\right) & A_{2} \\
A_{2} & \left(A_{0}+A_{1}\right)
\end{array}\right) .
$$

Conventions involving the two-component real Grassmann variable (Majorana) fields $\theta_{i}$ and $\lambda_{i}$ are given below in terms of $\theta$ with corresponding formulas for all such anticommuting variables. The derivative with respect to $\theta_{i}$ is defined through the Taylor expansion formula

$$
f(\theta+\delta \theta)=f(\theta)+\delta \theta_{i} \frac{\partial}{\partial \theta_{i}} f(\theta) .
$$

Hence the derivative is given by

$$
\frac{\partial}{\partial \theta_{i}} \theta_{j}=\delta_{i j}
$$

In a similar manner the conjugate Majorana spinor $\bar{\theta}_{i}$ is defined as

$$
\bar{\theta}_{i} \equiv\left(\gamma^{0}\right)_{i j} \theta_{j}=C_{i j} \theta_{j} .
$$

[For complex spinors (matter fields), $\psi_{i}$, the adjoint is defined as usual $\bar{\psi}=\psi^{\dagger} \gamma^{0}$.] The derivative with respect to $\bar{\theta}$ is defined analogously

$$
\frac{\partial}{\partial \bar{\theta}_{i}} \bar{\theta}_{j}=\delta_{i j}
$$

In other words

$$
\frac{\partial}{\partial \bar{\theta}_{i}}=-\left(\gamma^{0}\right)_{i j} \frac{\partial}{\partial \theta_{j}}
$$

so that the mixed derivative formulas are

$$
\begin{aligned}
& \frac{\partial}{\partial \bar{\theta}_{i}} \theta_{j}=-\left(\gamma^{0}\right)_{i j} \\
& \frac{\partial}{\partial \theta_{i}} \bar{\theta}_{j}=-\left(\gamma^{0}\right)_{i j} .
\end{aligned}
$$

Consequently the product of spinors

$$
\left(\bar{\theta}_{i} \psi_{i}\right)^{\dagger}=\bar{\theta}_{i} \psi_{i} .
$$

Further

$$
\theta_{i} \theta_{j}=\frac{1}{2} \bar{\theta} \theta \gamma_{i j}^{0}
$$

which leads to

$$
\begin{gathered}
\bar{\theta} \gamma^{m} \theta=0 \\
\bar{\theta} \gamma^{m} \gamma^{n} \theta=\bar{\theta} \theta \eta^{m n} \\
\bar{\theta} \gamma^{m} \gamma^{n} \gamma^{r} \theta=i \bar{\theta} \theta \epsilon^{m n r} .
\end{gathered}
$$
as

Projectors can be defined in terms of the $D=3$ vector $v^{m}$

$$
\begin{aligned}
& P_{v T}^{m n}=\left(\eta^{m n}-\frac{v^{m} v^{n}}{v^{2}}\right) \\
& P_{v L}^{m n}=\frac{v^{m} v^{n}}{v^{2}} .
\end{aligned}
$$

[1] S. R. Coleman, J. Wess, and B. Zumino, Phys. Rev. 177, 2239 (1969); C. G. Callan, S. R. Coleman, J. Wess, and B. Zumino, ibid. 177, 2247 (1969).

[2] D. V. Volkov, Sov. J. Part. Nucl. 4, 3 (1973); V. I. Ogievetsky, in Proceedings of the Xth Winter School of Theoretical Physics in Karpacz (Universitas Wratislaviensis, Wroclaw, 1974), Vol. 1, p. 227.

[3] E. A. Ivanov and V. I. Ogievetsky, Teor. Mat. Fiz. 25, 164 (1975).

[4] P. A. Dirac, Proc. R. Soc. London A268, 57 (1962).

[5] Y. Nambu, Phys. Rev. D 10, 4262 (1974).

[6] T. Goto, Prog. Theor. Phys. 46, 1560 (1971).

[7] M. Born and L. Infeld, Proc. R. Soc. London A144, 425 (1934).

[8] P. C. West, J. High Energy Phys. 02, 024 (2000).

[9] J. Hughes, J. Liu, and J. Polchinski, Phys. Lett. B 180, 370 (1986).

[10] J. Hughes and J. Polchinski, Nucl. Phys. B278, 147 (1986).

[11] M. J. Duff, Fields, Strings and Duality, TASI'96 Proceedings (World Scientific, Singapore, 1997), hep-th/9611203.

[12] P. C. West, hep-th/9811101.
[13] J. Bagger and A. Galperin, Phys. Lett. B 336, 25 (1994); Phys. Rev. D 55, 1091 (1997); hep-th/9810109.

[14] J. A. de Azcarraga, J. P. Gauntlett, J. M. Izquierdo, and P. K. Townsend, Phys. Rev. Lett. 63, 2443 (1989); E. R. Abraham and P. K. Townsend, Nucl. Phys. B351, 313 (1991); M. Cvetic, F. Quevedo, and S. J. Rey, Phys. Rev. Lett. 67, 1836 (1991); G. R. Dvali and M. A. Shifman, Phys. Lett. B 396, 64 (1997); 407, 452(E) (1997); A. Kovner, M. A. Shifman, and A. Smilga, Phys. Rev. D 56, 7978 (1997); A. Ritz, M. Shifman, and A. Vainshtein, ibid. 66, 065015 (2002); D. Binosi and T. ter Veldhuis, Phys. Lett. B 476, 124 (2000); M. Naganuma, M. Nitta, and N. Sakai, Phys. Rev. D 65, 045016 (2002); M. Naganuma and M. Nitta, Prog. Theor. Phys. 105, 501 (2001).

[15] E. Ivanov and S. Krivonos, Phys. Lett. B 453, 237 (1999); S. Bellucci, E. Ivanov, and S. Krivonos, ibid. 482, 233 (2000); S. Bellucci, E. Ivanov, and S. Krivonos, Nucl. Phys. B (Proc. Suppl.) 102, 26 (2001).

[16] E. Ivanov, Theor. Math. Phys. 129, 1543 (2001) [Theor. Math. Phys. 129, 278 (2001)]; E. Ivanov, in New Development in Fundamental Interaction Theories, edited by Jerzy Lukierski and Jakub Rembielinski, AIP Conf. Proc. 589 (AIP, Melville, NY, 2001), p. 61; hep-th/0002204. 
[17] See, for example, D. P. Sorokin, Phys. Rep. 329, 1 (2000), and references therein.

[18] B. Chibisov and M. A. Shifman, Phys. Rev. D 56, 7990 (1997); ibid. 58, 109901(E) (1998).

[19] Y. Sakamura, hep-th/0207159.

[20] D. V. Volkov and V. P. Akulov, JETP Lett. 16, 438 (1972).

[21] M. J. Duff and J. X. Lu, Nucl. Phys. B390, 276 (1993); P. K. Townsend, Phys. Lett. B 373, 68 (1996); C. Schmidhuber, Nucl. Phys. B467, 146 (1996).

[22] M. Aganagic, C. Popescu, and J. H. Schwarz, Nucl. Phys.
B495, 99 (1997).

[23] X. R. Hou, A. Losev, and M. A. Shifman, Phys. Rev. D 61, 085005 (2000); R. Hofmann and T. ter Veldhuis, ibid. 63, 025017 (2001); N. Maru, N. Sakai, Y. Sakamura, and R. Sugisaka, Phys. Lett. B 496, 98 (2000); N. Maru, N. Sakai, Y. Sakamura, and R. Sugisaka, Nucl. Phys. B616, 47 (2001); N. Sakai and R. Sugisaka, Phys. Rev. D 66, 045010 (2002).

[24] T. E. Clark and S. T. Love, Phys. Rev. D 54, 5723 (1996); ibid. 63, 065012 (2001). 